\title{
Impact of Case Volumes on the Outcomes of Percutaneous Nephrolithotomy
}

\author{
Dedan Opondo ${ }^{a}$, Ahmet Tefekli ${ }^{b}$, Tarik Esen ${ }^{b}$, Gaston Labate ${ }^{c}$, Kandasami Sangam $^{d}$, \\ Antonello De Lisa ${ }^{e}$, Hemendra Shah ${ }^{f}$, Jean de la Rosette ${ }^{a, *}$, \\ on behalf of the CROES PCNL study group
}

${ }^{a}$ Department of Urology, AMC University Hospital, Amsterdam, The Netherlands; ${ }^{\mathrm{b}}$ American Hospital, Istanbul, Turkey; ${ }^{\mathrm{c}}$ Urosalud, Buenos Aires, Argentina;

${ }^{\mathrm{d}}$ Department of Urology, Vedanayagam Hospital and Postgraduate Institute, Tamilnadu, India; ${ }^{\mathrm{e}}$ Urological Clinic, University of Cagliari, Cagliari, Italy; ${ }^{\mathrm{f}}$ Department of Urology, RG Stone Urology and Laparoscopy Hospital, Mumbai, India

\section{Article info}

\section{Article history:}

Accepted March 7, 2012

Published online ahead of

print on March 15, 2012

\section{Keywords:}

Case volume

Complications

Operating time

Outcomes

PCNL

Stone free

Urinary stones

\begin{abstract}
Background: Previous studies have demonstrated relationships between case volumes and outcomes in surgery. Little is known about the impact of case volumes on the outcomes of percutaneous nephrolithotomy (PCNL).

Objective: To investigate the influence of case volumes on the efficacy and safety outcomes of PCNL.

Design, setting, and participants: From November 2007 to December 2009, prospective data were collected by the Clinical Research Office of the Endourological Society from consecutive patients over a 1-yr period in 96 centers globally. Data of 3933 patients in the Global PCNL study database were included in this study.

Outcome measurements and statistical analysis: Patients were divided into low- and high-volume groups based on the median annual case volume of their respective treatment center. Preoperative characteristics and outcomes were compared between the two groups. Case volume was treated as a continuous variable. The relationship between case volume and stone-free (SF) rate, complication rate, and duration of hospital stay was explored using multivariate regression analysis.

Results and limitations: SF rates were higher in high-volume centers (82.5\% vs 75.1\%; $p$ value $<0.001$ ). Complication rates were lower in high-volume centers ( $15.9 \%$ vs $21.7 \%$; $p$ value 0.002 ), whereas the mean (standard deviation [SD]) duration of stay was shorter in high-volume centers (3.4 [2.6] vs 4.9 [3.7] d; $p$ value $<0.001$ ). SF rate increased with case volume, whereas complication rate and duration of stay diminished with increasing case volumes after adjusting for stone burden, urine culture status, American Society of Anesthesiologists score, and the presence of staghorn stones. The highest SF rates were observed in centers with $>120$ cases per year.

Conclusions: Centers that perform high numbers of PCNLs per year achieve better results. Both the efficacy and safety outcomes of PCNL improve with the number of surgeries performed in a given center per year.
\end{abstract}

(C) 2012 European Association of Urology. Published by Elsevier B.V. All rights reserved.

* Corresponding author. Department of Urology, AMC University Hospital, Meibergdreef 9 , 1105 AZ Amsterdam Z-O, The Netherlands. Tel. +31 20 5666030; Fax: +31 205669585. E-mail address: J.J.delaRosette@amc.uva.nl (J. de la Rosette). 


\section{Introduction}

Several research studies have been conducted to examine the effect of case volume on outcomes in high-risk surgical procedures $[1,2]$. It has been observed that surgical centers that perform high volumes of high-risk procedures achieve superior outcomes as compared with the lower volume centers, probably due to accrued surgical and institutional experience $[3,4]$.

This observation has also been described in urologic procedures. Studies that investigated the relationship between case volumes and outcomes in patients who undergo radical prostatectomy have demonstrated that centers with a high surgical volume tend to achieve better morbidity and mortality outcomes [5]. Similarly, Rioja et al. [6] and de la Rosette et al. [7] showed that the outcomes of percutaneous nephrolithotomy (PCNL) and ureterorenoscopy performed in a dedicated setting were superior to a nondedicated setting.

PCNL is the most invasive and technically complex contemporary method of managing renal stones [8]. Despite its low risk for perioperative mortality [9], PCNL has undergone spontaneous regionalization in the United States to high-volume, high-throughput teaching hospitals unlike other complex surgical procedures such as esophagectomy or pancreatectomy for which selective referral of high-risk cases was implemented as a policy [10]. In this study, we investigated the influence of case volume on the efficacy and safety outcomes in PCNL.

\section{Patients and methods}

\subsection{Data source}

From November 2007 to December 2009, prospective data were collected by the Clinical Research Office of the Endourological Society (CROES) from 5803 consecutive patients who were treated over a 1-yr period in 96 centers globally [11].

We included all adult patients who were enrolled in the Global PCNL study. Patients from centers with $<10$ cases of PCNL per year were excluded because of statistical reasons in the context of multiple covariates. Patients with a history of prior PCNL or open surgery were not included to avoid bias due to the impact of prior treatment on stonefree (SF) status.

\subsection{Estimation of annual case volume per center}

We estimated the annual case volume per center using the number of reported cases divided by the reported period of inclusion adjusted to $365 \mathrm{~d}$ as shown in this formula:

\section{Estimated case $=$ Reported cases per center volume per center $=\frac{\text { Reported inclusion period }}{\text { Repos }} \times 365$}

\subsection{Comparison of low- and high-volume centers}

We assigned the estimated case volume to each patient as an attribute representing the caseload of the center where the patient underwent treatment. A median of the estimated case volume was calculated from all included patients. Subsequently, the median case volume was used to divide the patients into two groups: those operated on in low-volume and high-volume centers, respectively. Patient characteristics, operative characteristics, and outcomes were calculated for both groups. Means and standard deviations were used for continuous variables; percentages were used for categorical variables.

Statistical tests of significance were performed on the baseline comparison of the low-volume versus the high-volume centers. The Student $t$ test was used for continuous variables; chi-square tests were used for the categorical variables.

\subsection{Relationship between case volumes and outcomes of percutaneous nephrolithotomy}

Multivariate regression models were built to explore the relationship between case volume and SF rates, the frequency and severity of complications, and the duration of the postoperative stay. SF status was assessed by ultrasonography, plain x-ray, or computed tomography (CT), depending on the policy of each contributing center. The multivariate regression analysis for the outcome variables was adjusted for the presence of staghorn stones, urine culture status, stone burden, and American Society of Anesthesiologists (ASA) score. Case volume was considered as a continuous variable in these regression models and was analyzed to explore nonlinear relationships with the outcome variables. The multivariate model for the relationship between case volume and treatment success was adjusted for the differences in the method used for assessing SF status.

All statistical analysis was performed using R-statistical programming software v.2.12.2. The level of statistical significance was set at 0.05 .

\section{Results}

\subsection{Comparison of high- and low-volume groups}

A total of 3933 adults were included in this study. Median (range) estimated operating case volume was 77 (10-289) PCNLs per year. Thirteen centers were classified as highvolume centers ( $>77$ cases per year), and 58 centers were classified as low-volume centers ( $<77$ cases per year). No statistically significant differences were observed in the age, sex, and comorbidity patterns between patients in the two groups. There were more ASA I patients in the high-volume group. The low-volume group had more patients in ASA II-IV as shown in Table 1. Staghorn stones were present in $29.7 \%$ and $23.0 \%$ ( $p$ value $<0.001$ ) of patients in the low- and high-volume groups, respectively. Stone burden was slightly higher among the patients in the high-volume group ( $p$ value 0.084 ).

The higher volume group had more patients in the prone position compared with the supine position. Lower pole access was the most common approach in both groups. Operation time was shorter in the high-volume group. For intraoperative complications, the perforation rate was statistically significantly lower in the high-volume group. Failed access and hydrothorax rates were not statistically significantly different between the two groups, although both rates were lower in the high-volume group. Similarly, reported bleeding rates and a drop in hemoglobin concentration were also lower in the high-volume group, as shown in Table 2.

\subsection{Case volume versus stone-free rates}

SF rates were significantly higher in high-volume centers. Multivariate analysis showed that SF rates improved with 
Table 1 - Baseline characteristics of patients and renal stones included in the study

\begin{tabular}{|c|c|c|c|}
\hline Characteristic & $\begin{array}{l}\text { Low } \\
\text { volume } \\
n=1994\end{array}$ & $\begin{array}{l}\text { High } \\
\text { volume } \\
n=1939\end{array}$ & $p$ value \\
\hline No. of centers & 58 & 13 & - \\
\hline No. of PCNL cases, mean & 33.8 & 149.2 & - \\
\hline Age, yr, mean (SD) & $52.2(14.5)$ & $49.0(14.0)$ & $<0.001$ \\
\hline \multicolumn{4}{|l|}{ Sex } \\
\hline Male, \% & 56.8 & 57.3 & \multirow{2}{*}{0.452} \\
\hline Female, \% & 43.2 & 42.7 & \\
\hline \multicolumn{4}{|l|}{ Comorbidities } \\
\hline $\mathrm{DM}, \%$ & 15.0 & 12.1 & 0.014 \\
\hline CVD, \% & 24.9 & 22.0 & 0.048 \\
\hline Anticoagulation, $\%$ & 6.8 & 4.6 & 0.006 \\
\hline \multicolumn{4}{|l|}{ ASA classification } \\
\hline ASA I, \% & 50.3 & 63.0 & \multirow{4}{*}{$<0.001$} \\
\hline ASA II, \% & 37.0 & 29.3 & \\
\hline ASA III, \% & 11.6 & 6.9 & \\
\hline ASA IV, \% & 1.1 & 0.8 & \\
\hline Staghorn stones, \% & 29.7 & 23.0 & $<0.001$ \\
\hline $\begin{array}{l}\text { Stone burden, } \mathrm{mm}^{2} \text {, } \\
\text { mean }(\mathrm{SD})\end{array}$ & $393.8(382.5)$ & $414.0(360.4)$ & 0.084 \\
\hline \multicolumn{4}{|c|}{$\begin{array}{l}\text { PCNL = percutaneous nephrolithotomy; } \mathrm{SD}=\text { standard deviation; } \\
\mathrm{DM}=\text { diabetes mellitus; } \mathrm{CVD}=\text { cardiovascular disease; } \mathrm{ASA}=\text { American } \\
\text { Society of Anesthesiologists (score). }\end{array}$} \\
\hline
\end{tabular}

increasing caseload with a peak at 120 cases per year as shown in Figure 1a. This finding was similarly observed again after adjusting stone burden, presence of staghorn stones, and the method used for checking SF status. Highest SF rates were achieved with estimated stone volume between 100 and $300 \mathrm{~mm}^{2}$. SF rates had an inverse linear

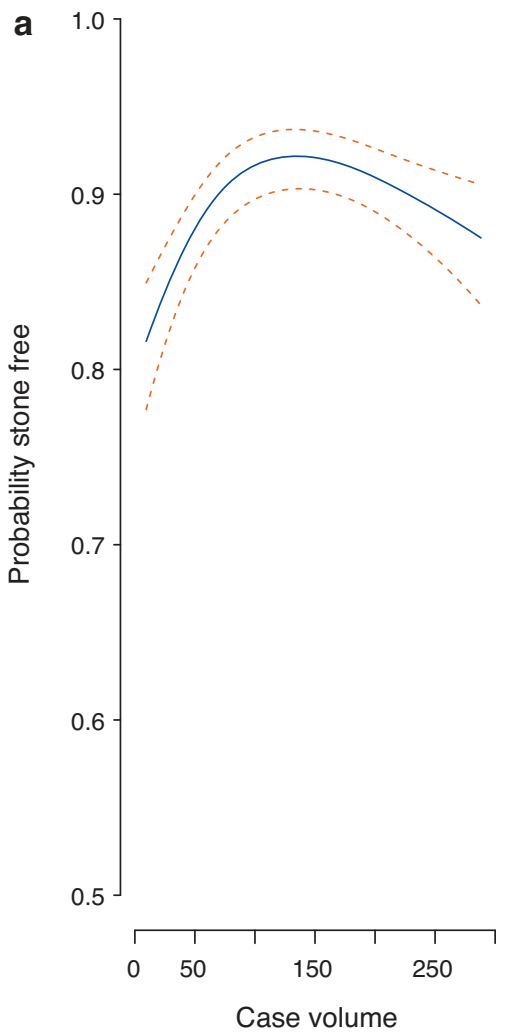

Table 2 - Operative characteristics

\begin{tabular}{|c|c|c|c|}
\hline Characteristic & $\begin{array}{l}\text { Low } \\
\text { volume } \\
n=1994\end{array}$ & $\begin{array}{l}\text { High } \\
\text { volume } \\
n=1939\end{array}$ & $p$ value \\
\hline \multicolumn{4}{|l|}{ Patient position } \\
\hline Supine, \% & 28.2 & 12.3 & \multirow[b]{2}{*}{$<0.001$} \\
\hline Prone, \% & 71.8 & 87.7 & \\
\hline \multicolumn{4}{|l|}{ Renal puncture site } \\
\hline Upper pole, \% & 10.1 & 7.9 & \multirow{3}{*}{$<0.001$} \\
\hline Middle pole, \% & 15.1 & 16.6 & \\
\hline Lower pole, \% & 70.3 & 66.0 & \\
\hline Multiple poles, \% & 4.5 & 9.5 & \\
\hline \multicolumn{4}{|l|}{ Location of access } \\
\hline Above 11 th rib, $\%$ & 1.2 & 1.5 & \multirow{3}{*}{0.158} \\
\hline Above 12 th rib, $\%$ & 14.4 & 16.6 & \\
\hline Below 12th rib, \% & 84.4 & 81.9 & \\
\hline Postoperative stent, \% & 40.1 & 44.2 & 0.005 \\
\hline Postoperative nephrostomy, \% & 89.5 & 93.9 & $<0.001$ \\
\hline Operating time, min, mean (SD) & $90.2(48.4)$ & $72.0(39.4)$ & $<0.001$ \\
\hline \multicolumn{4}{|l|}{ Complications } \\
\hline Failed access, $\%$ & 1.8 & 1.4 & 0.266 \\
\hline Perforation, \% & 4.4 & 2.2 & $<0.001$ \\
\hline Hydrothorax, \% & 1.6 & 1.4 & 0.536 \\
\hline \multicolumn{4}{|l|}{ Blood loss parameters } \\
\hline Reported bleeding, \% & 9.0 & 5.5 & $<0.001$ \\
\hline $\begin{array}{l}\text { Change in hemoglobin, } \\
\mathrm{mg} / \mathrm{dl} \text {, mean }(\mathrm{SD})\end{array}$ & $3.9(3.2)$ & $3.5(3.4)$ & 0.004 \\
\hline Blood transfusion rate ${ }^{*}, \%$ & 5.24 & 3.40 & $<0.001$ \\
\hline $\begin{array}{l}\text { SD = standard deviation. } \\
\text { Blood transfusion rates are adju } \\
\text { concentration. }\end{array}$ & ed for bas & e hemoglobin & \\
\hline
\end{tabular}

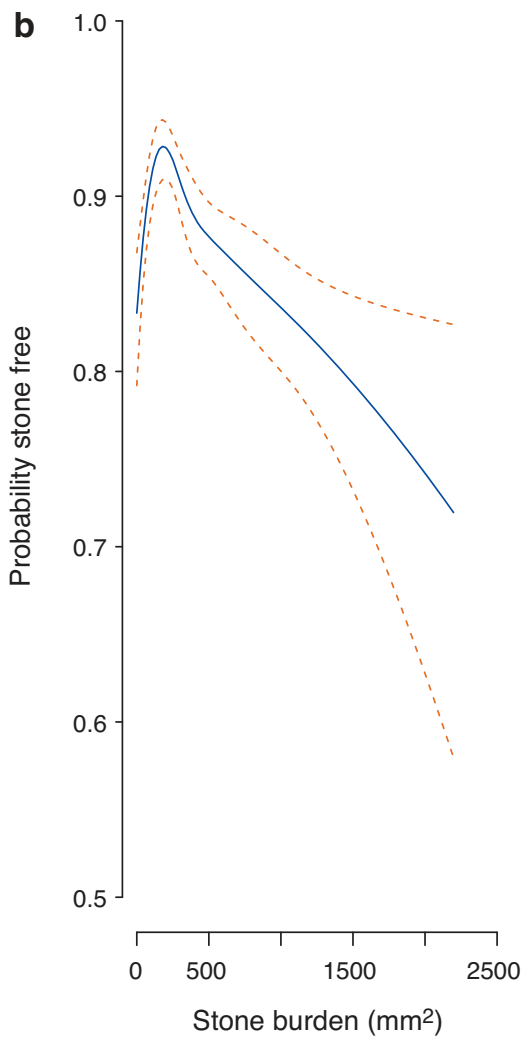

Fig. 1 - Relationship between case volume and stone-free (SF) rates. (a) SF rates increase with caseload and peak at approximately 120 cases per year. A further increase in case volumes is associated with a gradual decline in SF rate. (b) Adjusted relationship between stone burden and SF rate. 
relationship beyond the estimated stone volume of $500 \mathrm{~mm}^{2}$. Notably, stones with an estimated volume $<100 \mathrm{~mm}^{2}$ tended to have lower SF rates as shown in Figure 1b. Patients with staghorn stones had lower SF rates. The multivariate model revealed that CT scanning (81\%; 95\% confidence interval [CI], 76-85) detected more residual stones compared with ultrasound (86\% [95\% CI, 82-89\%]) and kidney, ureters, bladder (KUB) (x-ray) $(88 \%$ [95\% CI, 88-92). With CT scan as the reference method for SF evaluation, ultrasound and KUB SF rates were statistically significant with $p$ values of 0.0261 and $<0.001$, respectively.

Retreatment rate was lower in the high case volume group compared with the low case volume group.

\subsection{Case volume versus incidence and severity of complications}

Patients in the high case volume group had fewer minor and fewer major complications when compared with those in the low case volume group, as shown in Table 3. Multivariate analysis showed that the incidence of complications after PCNL was lower with an increasing case volume with a nadir at 120 cases per year, adjusted for stone burden, the presence of staghorn stones, urine culture, and the ASA score as shown in Figure 2a. Similarly, the severity
Table 3 - Postoperative outcomes

\begin{tabular}{lccr}
\hline Characteristic & $\begin{array}{c}\text { Low } \\
\text { volume } \\
n=1994\end{array}$ & $\begin{array}{c}\text { High } \\
\text { volume } \\
n=1939\end{array}$ & $p$ value \\
& 74.8 & 82.6 & $<0.001$ \\
\hline Stone-free rate, \%, all modalities & 15.3 & 12.2 & 0.002 \\
Retreatment rate, \% & $4.9(3.7)$ & $3.4(2.6)$ & $<0.001$ \\
Length of hospital stay, mean (SD) & & & \\
Complications (within 30 d) & 21.7 & 15.9 & $<0.001$ \\
$\quad$ Total complication rate, \% & 17.7 & 12.2 & \\
$\quad$ Minor complications, \%, & & & $<0.001$ \\
$\quad$ Clavien I and II & 4.0 & 3.7 & \\
$\quad$ Major complications, \% Clavien III-V & & & \\
Clavien categories & 13.0 & 7.3 & \\
$\quad$ Clavien I, \% & 4.7 & 4.9 & \\
$\quad$ Clavien II, \% & 2.2 & 2.5 & $<0.001$ \\
Clavien IIIA, \% & 1.3 & 1.0 & \\
$\quad$ Clavien IIIB, \% & 0.4 & 0.2 & \\
Clavien IVA, \% & 0.1 & 0.1 & \\
$\quad$ Clavien IVB, \% & 0.1 & 0.0 & \\
$\quad$ Clavien V, \% & & & \\
\hline SD = standard deviation. & &
\end{tabular}

of complications per center, measured by mean Clavien score, was reduced with increasing case volume with a nadir at 120 cases per year as shown in Figure $2 \mathrm{~b}$. The occurrence of complications increased with the estimated
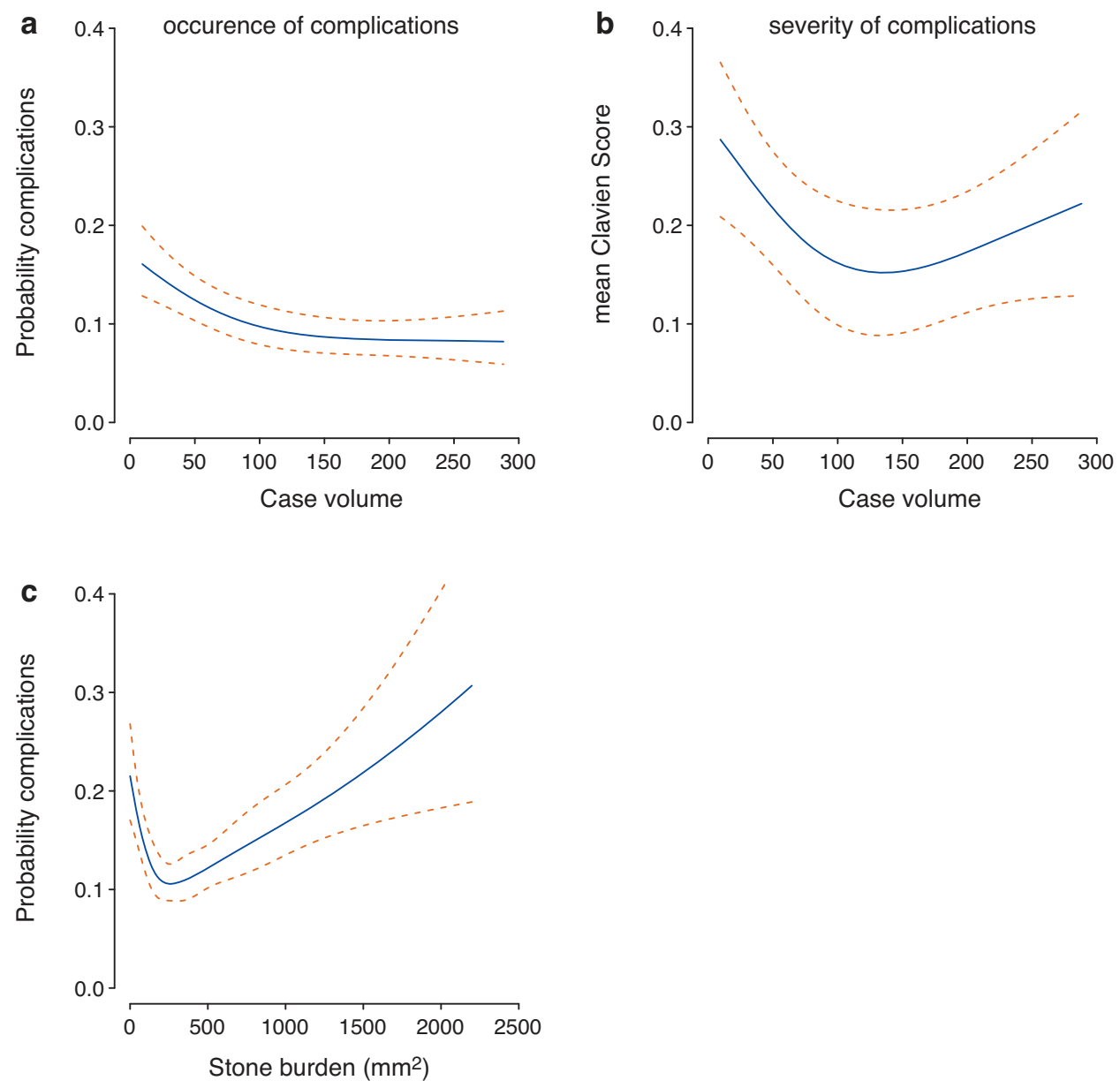

Fig. 2 - Relationship between case volume and complications adjusting for stone burden, presence of staghorn stones, American Society of Anesthesiologists (ASA) score, and urine culture status. (a) Decrease in the occurrence of any postoperative complication; (b) decrease in the severity of postoperative complications with increased case volume; (c) relationship between the occurrence of postoperative complications and stone burden. 

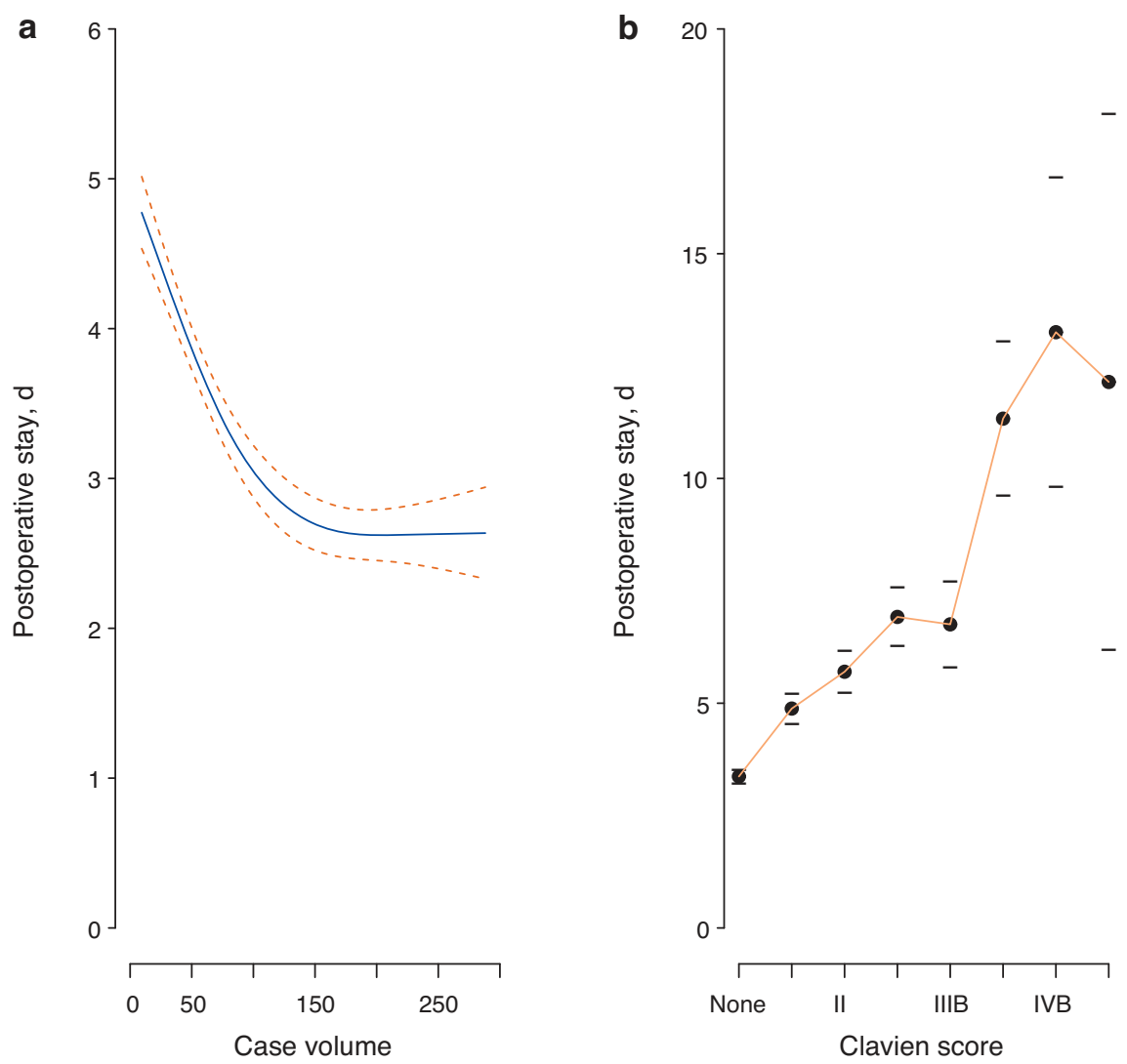

Fig. 3 - Relationship between case volume and duration of postoperative stay adjusting for severity of complication (Clavien score) and the presence of staghorn stones. (a) Decrease in postoperative stay with increase in case volume. No further decrease in postoperative stay is observed with case volumes greater than approximately 140 cases per year. (b) The adjusted relationship between postoperative complications on duration of postoperative stay.

stone volume. The lowest complication rates were observed with stones between 100 and $500 \mathrm{~mm}^{2}$. Stones with an estimated volume $<100 \mathrm{~mm}^{2}$ tended to have an inverse relationship with the frequency of complications (Fig. 2c). Patients with staghorn stones had more complications than those without. ASA scores were positively associated with the risk of postoperative complications. Patients with a preoperative positive urine culture had a greater chance of developing postoperative complications.

\subsection{Case volume versus duration of hospital stay}

Hospital stay was shorter in the high case volume group. On multivariate analysis the duration of postoperative stay was shorter with an increase in the case volume with a nadir at 140 cases per year as shown in Figure 3a. Thereafter there was no statistically significant difference in the reduction of postoperative stay and an increase in case volume. This observation was true after adjusting for the severity of postoperative complications and the presence of staghorn stones. Patients with more severe postoperative complications stayed longer than those who had minor complications (Fig. 3b). Similarly, patients with staghorn stones had a longer hospital stay. A high ASA score was indirectly associated with a longer hospital stay because higher ASA scores resulted in more complications and high Clavien scores that were associated with a longer hospital stay.

\section{Discussion}

We showed in this study that centers performing large numbers of PCNL per year achieve higher SF rates, lower complication rates, and shorter hospital stays. High case volumes are thus associated with better quality outcomes of PCNL surgery.

We speculate that centers performing large numbers of PCNLs are likely to achieve higher levels of team efficiency leading to better coordination during PCNL. Similar results were seen in studies investigating surgical outcomes in high-risk surgeries [12]. Wilt et al. showed that the concentration of high-risk surgical procedures in dedicated centers resulted in superior outcomes [13]. We noted that further increases in case volumes were associated with a decline in quality outcomes, however. SF rates decreased and the severity of documented complications gradually increased with $<120$ and $<140$ cases per year, respectively. We speculate that centers performing very high case volumes are likely to have surgeons with difference experience levels. Some of the centers could also be teaching centers with trainees whose complication rates might be higher. It is also possible that these high-volume centers received referred cases that may be surgically more complex. Such selective referrals of patients to a highvolume center may account for the lower performance of centers with very high case volumes. 
Our results documented that both safety and efficacy outcomes improve with increasing case volume. Previous studies showed that centers that perform better on one outcome indicator tend to perform well on others [4]. In our study we clearly showed that high-volume centers perform significantly better than low-volume centers in terms of SF rates, complication rates, and hospital stay.

Based on Global PCNL data, we estimate that centers performing two to three PCNLs per week achieve the best outcomes. However, it remains difficult to set a specific cutoff for the minimal number of PCNLs that a center should perform to attain optimal results. Distributions of centers that perform PCNL vary from country to country. Likewise, base populations differ from region to region, so other factors that affect access to percutaneous stone treatment have to be considered when determining the threshold for defining centers as high volume or low volume. Although initiatives to facilitate referral of patients to high-volume hospitals have the potential to improve outcomes in some medical conditions [14], we believe that each region/ country should determine their referral policies based on local case volume patterns and experience. On the contrary, some authors have suggested that low-volume centers may improve their urologic outcomes by identifying factors that contribute to high quality at high-volume centers and implementing them in their own practices [15].

This study was inherently limited by the heterogeneity of centers and surgeons that participated in the study. We did not collect data on the number and experience of the individual surgeons in the centers included in the study. Although data were collected prospectively, the nature of the study design showed several further interesting findings. Staghorn stones constituted a higher percentage of cases in the low-volume group, in which supine as well as tubeless approaches were surprisingly more common. These findings probably need to be investigated in more detail.

To our knowledge, this was the first study to investigate explicitly the relationship between case volume and the outcome of PCNL. We used guidelines for executing reliable volume-outcome studies as described by Halm et al. and achieved a total score of 16 of 18 [16]. Our multicenter study evaluated the outcomes of PCNL in terms of SF and complication rates as well as length of hospital stay simultaneously while adjusting for known confounders of the outcomes. We believe that no single outcome measure can ideally represent the total quality of PCNL. Furthermore, we analyzed the case volume as a continuous variable that gives a clearer relationship with outcomes instead of using arbitrary cut-off volumes. Hong et al. recommended this approach in their guideline for reporting the population-based volume-outcome relationship in surgery [17]. And our data revealed that the most satisfactory results were achieved in centers performing $>120$ cases per year.

\section{Conclusions}

High-volume centers attain higher SF rates with lower complications and shorter hospital stay compared with low-volume centers. These efficacy and safety outcomes for PCNL improve with an increase in the case volume.

Author contributions: Jean de la Rosette had full access to all the data in the study and takes responsibility for the integrity of the data and the accuracy of the data analysis.

Study concept and design: de la Rosette, Esen, Tefekli.

Acquisition of data: Opondo, Tefekli, Esen, Labate, Sangam, De Lisa, Shah, de la Rosette.

Analysis and interpretation of data: Opondo, de la Rosette.

Drafting of the manuscript: Opondo, de la Rosette.

Critical revision of the manuscript for important intellectual content: Opondo, Tefekli, Esen, Labate, Sangam, De Lisa, Shah, de la Rosette.

Statistical analysis: Opondo.

Obtaining funding: de la Rosette.

Administrative, technical, or material support: Opondo, Tefekli, Esen, Labate, Sangam, De Lisa, Shah, de la Rosette.

Supervision: de la Rosette.

Other (specify): None

Financial disclosures: The corresponding author certifies that all conflicts of interest, including specific financial interests and relationships and affiliations relevant to the subject matter or materials discussed in the manuscript (eg, employment/affiliation, grants or funding, consultancies, honoraria, stock ownership or options, expert testimony, royalties, or patents filed, received, or pending), are the following: None.

Funding/Support and role of the sponsor: Olympus helped design and conduct the study.

\section{References}

[1] Birkmeyer JD, Stukel TA, Siewers AE, Goodney PP, Wennberg DE, Lucas FL. Surgeon volume and operative mortality in the United States. N Engl J Med 2003;349:2117-27.

[2] Goodney PP, Stukel TA, Lucas FL, Finlayson EV, Birkmeyer JD. Hospital volume, length of stay, and readmission rates in high-risk surgery. Ann Surg 2003;238:161-7.

[3] Seitz C, Desai M, Hacker A, et al. Incidence, prevention, and management of complications following percutaneous nephrolitholapaxy. Eur Urol 2012;61:146-58.

[4] Hewitt M, Petitti D, editors. Interpreting the volume-outcome relationship in the context of cancer care. Washington, DC: National Academy Press; 2001.

[5] Bianco Jr FJ, Riedel ER, Begg CB, Kattan MW, Scardino PT. Variations among high volume surgeons in the rate of complications after radical prostatectomy: further evidence that technique matters. J Urol 2005;173:2099-103.

[6] Rioja J, Mamoulakis C, Sodha H, Suwijn S, Laguna P, de la Rosette J. A plea for centralized care for ureteroscopy: results from a comparative study under different conditions within the same center. J Endourol 2011;25:425-9.

[7] de la Rosette JJ, Zuazu JR, Tsakiris P, et al. Prognostic factors and percutaneous nephrolithotomy morbidity: a multivariate analysis of a contemporary series using the Clavien classification. J Urol 2008;180:2489-93.

[8] Lingeman JE. Percutaneous nephrolithotomy: burden or opportunity. J Urol 2006;176:14.

[9] Labate G, Modi P, Timoney A, et al., CROES PCNL Study Group. The percutaneous nephrolithotomy global study: classification of complications. J Endourol 2011;25:1275-80.

[10] Morris DS, Taub DA, Wei JT, Dunn RL, Wolf Jr JS, Hollenbeck BK. Regionalization of percutaneous nephrolithotomy: evidence for the increasing burden of care on tertiary centers. J Urol 2006;176:242-6. 
[11] de la Rosette J, Assimos D, Desai M, et al. The Clinical Research Office of the Endourological Society Percutaneous Nephrolithotomy Global Study: indications, complications, and outcomes in 5803 patients. J Endourol 2011;25:11-7.

[12] Nuttall M, van der Meulen J, Phillips N, et al. A systematic review and critique of the literature relating hospital or surgeon volume to health outcomes for 3 urological cancer procedures. J Urol 2004;172: 2145-52.

[13] Wilt TJ, Shamliyan TA, Taylor BC, MacDonald R, Kane RL. Association between hospital and surgeon radical prostatectomy volume and patient outcomes: a systematic review. J Urol 2008;180: $820-8$.
[14] Dudley RA, Johansen KL, Brand R, Rennie DJ, Milstein A. Selective referral to high-volume hospitals: estimating potentially avoidable deaths. JAMA 2000;283:1159-66.

[15] Joudi FN, Konety BR. The impact of provider volume on outcomes from urological cancer therapy. J Urol 2005;174:432-8.

[16] Halm EA, Lee C, Chassin MR. Is volume related to outcome in health care? A systematic review and methodologic critique of the literature. Ann Intern Med 2002;137:511-20.

[17] Hong D, Tandan VR, Goldsmith CH, Simunovic M. Users' guide to the surgical literature: how to use an article reporting population-based volume-outcome relationships in surgery. Can J Surg 2002;45: 109-15. 